\title{
Percepción de carga del cuidado en cuidadores informales de personas con enfermedad mental en Bogotá
}

\author{
Perception of the burden of care in informal caregivers of persons with \\ mental illness in Bogotá
}

\begin{abstract}
Resumen
El artículo expone una investigación cualitativa de interés interpretativo; se buscó conocer la percepción sobre la labor de cuidado y síndrome de carga en cuidadores informales de personas mayores de 18 años con enfermedad mental atendidas en un centro de atención en Bogotá mediante un grupo focal y aplicación de entrevistas individuales no estructuradas. Para la interpretación de la información se abordan las categorías de carga estructural y carga individual. El cuidador informal es delegado con frecuencia por decisión familiar, ninguno cuenta con remuneración económica y su labor conlleva a que sus espacios sociales y laborales se reduzcan o anulen. Se presentan casos de cuidadores con enfermedades físicas las cuales deben sobrellevar simultáneamente con su labor produciendo tensiones a nivel emocional. Los resultados reflejan la pertinencia y necesidad de desarrollar acciones para el reconocimiento social y legal del cuidador junto espacios preventivos a enfermedades desencadenadas por su labor.
\end{abstract}

\section{Palabras clave}

Cuidador informal, cuidado, enfermedad mental, síndrome de carga del cuidador, carga estructural, carga individual.

\begin{abstract}
The article exposes a qualitative research of interpretative interest; we sought to know the perception about the work of care and burden syndrome in informal caregivers of people over 18 years of age with mental illness attended in a care center in Bogotá through a focus group and application of unstructured individual interviews. For the interpretation of the information, the categories of structural load and individual load are addressed. The informal caregiver is often delegated by family decision, no one has economic remuneration and their work leads to their social and labor spaces are reduced or canceled. There are cases of caregivers with physical diseases which must cope with their work simultaneously producing tensions on an emotional level. The results reflect the relevance and need to develop actions for the social and legal recognition of the caregiver together with preventive spaces for illnesses unleashed by their work.
\end{abstract}

\section{Keywords}

Informal caregiver, care, mental illness, caregiver burden syndrome, structural burden, individual burden.

\author{
Franci Camila Amezquita \\ Torres \\ <camilatorres.0311@gmail.com> \\ Universidad Pedagógica Nacional. \\ Colombia
}

Astrid Karime Moreno Cano <nogenuflectos@hotmail.com>

Universidad Colegio Mayor de Cundinamarca. Colombia

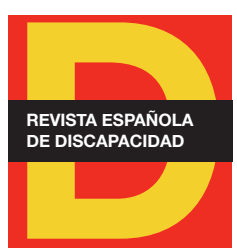

Para citar:

Amezquita, F. C y Moreno, A. K. (2019): "Percepción de carga del cuidado en cuidadores informales de personas con enfermedad mental en Bogotá". Revista Española de Discapacidad, 7 (II): 55-77.

Doi: <https://doi.org/10.5569/23405104.07.02.04>

Fecha de recepción: 11-07-2018 Fecha de aceptación: 30-10-2019 


\section{Introducción}

La definición de discapacidad inicialmente estuvo permeada por fundamentos religiosos, posteriormente pasa a ser vista desde una perspectiva científica-médica en la que se comprendía como la restricción o falta de la capacidad de un ser humano para ejecutar actividades en el margen de lo considerado "normal". En contraposición a este planteamiento aparece una perspectiva fundamentada por un modelo social que afirma que esta no es un resultado del individuo que la presenta, sino de las limitaciones impuestas por la sociedad que no permiten la respuesta óptima a sus necesidades e inclusión en la sociedad (Hernández, 2015).

Desde un enfoque biopsicosocial se afirma que la discapacidad se gesta a partir de un proceso relacional en el que emergen interacciones complejas entre una persona, sus limitaciones funcionales físicas, mentales o intelectuales y el ambiente en el que se encuentra (OMS, 2001). Asimismo, la Convención sobre los derechos de las personas con discapacidad la define como "un concepto que evoluciona y que resulta de la interacción entre las personas con deficiencias y aquellas barreras debidas a la actitud y al entorno que evitan su participación plena y efectiva en la sociedad, en igualdad de condiciones con los demás" (ONU, 2006: 2).

En cuanto a la enfermedad mental, se define dentro de esta categoría a la presencia de trastornos mentales como la esquizofrenia, trastornos psicóticos, trastorno afectivo bipolar, depresión, trastorno de la conducta alimentaria entre otros (Aretio, 2010) las cuales son clasificadas en el Manual Diagnóstico y Estadístico de los Trastornos Mentales DSM-5 (American Psychiatric Association, 2014) y la Clasificación Estadística Internacional de Enfermedades y Problemas relacionados con la Salud (CIE 10) (Organización Mundial de la Salud, 2001b).

La presencia de enfermedades mentales en una persona pueden generar tensiones y desequilibrios en ella y los miembros de su sistema familiar, por tal razón, no pueden concebirse como eventos que afectan de manera individual (Velasco y Sinibaldi, 2001), pues las características de cada diagnóstico pueden conllevar que el individuo que las padece presente disfuncionalidad social y laboral y requiera de acciones de cuidado, siendo esta actividad concebida por Watson (1979) como una labor orientada a "la satisfacción de necesidades humanas en el ámbito de la salud de un individuo, comunidad o población, como también integrativo y holístico, porque abarca los diferentes estratos de necesidades" (Citado por Urra et al., 2011: 18). También definido desde el amor y como una actitud (Boff, 2002) la cual se lleva a cabo desde la comprensión de la persona que se cuida, lo que refleja una relación ética (Gilligan, 1982), y de manera artesanal (De la Cuesta, 2004); desde otras posturas se aborda un cuidado desde procesos educativos (Noddings, 2005) o el cuidado médico y psicológico que requiere la persona cuidada.

Históricamente el cuidado ha sido visto como una práctica femenina donde se gestan interrogantes alrededor de la relación de cuidado, las implicaciones económicas, emocionales y físicas que atraviesa la cuidadora (Daly y Lewis, 2000) debido a la inequidad del cuidado (Vaquiro y Stiepovich, 2010) que ha conllevado a que sean víctimas de explotación económica al no reconocerse su labor (Martín, 2008). Esta situación se evidencia como producto de la normalidad social y del patriarcado que han llevado a una división de trabajo en el que las mujeres cuidan y los hombres se encuentran en escenarios laborales formales (Tobío, 2012), no obstante, las redes de apoyo pueden ser limitadas en aquellos casos de que la mujer es la única cuidadora y proveedora económica del hogar. 
Por otro lado, a nivel estadístico, en Bogotá (Colombia), la labor de cuidado predomina en las mujeres en un $91 \%$; un $55 \%$ corresponde a quienes tienen 51 años o más y un $28 \%$ corresponde a mujeres cuidadoras entre los 36 y 50 años. En cuanto al nivel educativo, un $48 \%$ de los cuidadores cuentan con primaria incompleta debido a dificultades económicas y un $92 \%$ no cuentan con una remuneración económica por la labor de cuidado realizada (Gómez et al., 2016). Lo anterior lleva a pensar que la mujer en ciclo vital de vejez presenta mayor vulnerabilidad por las enfermedades desencadenadas por el mismo transcurrir vital en el que se encuentra y que pueden entrar en estado crítico por el constante esfuerzo físico y mental generado en la labor de cuidado, de igual manera, la presencia de mujeres en edad productiva conlleva que sus metas personales en la construcción de proyecto de vida, en muchas ocasiones, deban ser dejadas de lado para responder a la labor de cuidado de manera permanente.

Se identifica que el cuidado es una labor delegada a la mujer, empero, los cambios de dinámica social, en los que se evidencia la migración rural-urbana y la vinculación de la mujer en el mundo laboral, han conllevado que el cuidado no se refleje como una actividad únicamente femenina (Moreno y Cárdenas, 2010). En este mismo sentido, se encuentran postulados que indican que la actividad de cuidado en la familia se comparte con otros miembros del sistema (Abellán y Esparza, 2010).

Dentro del carácter nacional orientado por la Ley 33 del 2009 se define cuidador informal como un miembro perteneciente a la familia de la persona en condición de discapacidad que presenta hasta un quinto grado de consanguinidad, tercero de afinidad o primero civil y quien asume una labor de cuidado constante en aras de atender las necesidades que su familiar no puede responder de manera independiente a causa de alguna discapacidad; resaltando que esta labor conlleva que este familiar cuidador no pueda desempeñarse en el mercado laboral. Este perfil se acerca a las definiciones abordadas por estudios adelantados alrededor del cuidador informal como es el caso de autores como Castro et al. (2009).

El cuidador se define a partir de dos perfiles, por un lado, los cuidadores formales, quienes poseen conocimientos médicos o técnicos para ejercer la labor (De la Cuesta, 2004); y, por otro, cuando una familia no puede acceder a este tipo de cuidado debido al costo económico, aparece la figura de los cuidadores informales, delegados por la familia y quienes realizan su actividad de atención de manera constante y sin remuneración, sin horario de descanso ni un reconocimiento legal de sus necesidades como persona (Aguas, 1999; De la Cuesta, 2004).

Ahora bien, la presencia del Estado a través de un modelo de bienestar ha buscado dar alcance al desarrollo y bienestar social en el que, desde palabras de Esping (2000) resulta necesario recordar que el núcleo familiar es un componente fundamental para un régimen de bienestar debido al ejercicio de cuidado que se presenta entre sus miembros para la permanencia de la estructura familiar; lo anterior lleva a un análisis de la visión dominante masculina dentro de los procesos de bienestar (Orloff, 1993, 1996; Sainsbury, 1996, 1999; Skocpol, 1992; entre otros). Además de lo anterior, se presenta una crítica al evidenciar que el individuo se encuentra mercantilizado y sus derechos son garantizados conforme su trabajo cuente con un grado de mercantilización, por lo cual, el cuidado familiar, que en su mayoría es femenino, no es reconocido como un trabajo mercantilizado.

Lo mencionado lleva a la construcción de un respaldo legal para el reconocimiento del cuidador informal en Colombia dado que no cuenta con un ingreso económico de su labor como tampoco con tiempo y medios 
para vincularse a una red laboral o académica. No obstante, pese a estos esfuerzos no se evidencia un avance; en la Ley 1392 de 2010 se hace visible la labor de cuidado, pero no se logra dentro de esta legitimar una remuneración económica para el cuidador, lo que conlleva a que se perpetúe su situación de inestabilidad en dicho aspecto. Por otro lado, desde el Proyecto de Ley 62 de 2014 se busca garantizar la manutención de personas que estén a cargo del cuidado de alguna persona con discapacidad, no obstante, este se encuentra inactivo desde el año 2016. Según la Agencia de Información Laboral-ESN (2016), a su vez, mediante la Resolución Número 005928 de 2016 emitida por el Ministerio de Salud y Protección Social, se establecen los requisitos para el reconocimiento y pago de la labor de cuidado mediante fallo de tutela.

Cabe resaltar que existe una política pública de discapacidad en la que se brindan beneficios económicos y de atención a la población adscrita, sin embargo, la figura del cuidador aparece como un actor secundario dentro de la relación de cuidado, las estrategias para su atención comprenden espacios de respiro que no pueden ser disfrutados por el cuidador de manera individual puesto que no cuenta con una red familiar o social que apoye en su labor de cuidado, lo que conduce a que su labor la realice ante la adversidad (De la Cuesta, 2004).

Otra tensión que afronta el cuidador son los dobles vínculos que emergen alrededor de la relación de cuidado (Bateson et al., 1956). El cuidador y la persona cuidada pueden recibir dos tipos de mensaje; por un lado, el cuidador puede percibir que su cuidado se requiere de manera incondicional pero recibe violencia verbal y/o física por parte de la persona cuidada y, por otro lado, la persona cuidada puede percibir que el cuidador le atiende desde su sentimiento de amor pero en los momentos de tensión debe recibir las emociones de tristeza e ira que pueda tener el cuidador informal; estas situaciones pueden conllevar a una relación de cuidado revestida de trato hostil, distante, de auto-anulación pero también de necesidad de mantenerla puesto que los dos actores inmersos en la relación de cuidado se sienten reconocidos por el otro.

Dichas situaciones pueden producir el síndrome de carga del cuidador, entendido por Zambrano y Ceballos (2007) como "estar quemado" e indica agotamiento mental y ansiedad frente al cuidado. También está relacionado con dificultades en la salud física, debido a una acumulación de estresores frente a los que el cuidador se encuentra desprovisto de estrategias adecuadas de afrontamiento para adaptarse a la situación" (p. 28); los estresores que puede sobrellevar el cuidador son incremento de gastos y energía, el cambio de conducta repentina de la persona cuidada, la dificultad de que se logre movilizar o en el acceso a espacios de ocio y fortalecimiento de relaciones sociales. Así mismo representa "la dificultad persistente de cuidar y los problemas físicos, psicológicos y emocionales que pueden estar experimentando o ser experimentados por miembros de la familia que cuidan a un familiar con incapacidad o algún deterioro" (Zambrano y Ceballos, 2007: 28) lo cual puede desencadenar en el cuidador problemas en su salud emocional y mental, principalmente depresión debido al estrés que produce su labor (Crespo y López, 2006) siendo un tema abordado como "estudio del estrés y de las reacciones emocionales" a nivel nacional e internacional (Palacios y Jiménez, 2008).

En relación a investigaciones adelantadas se encuentran estudios relacionados con el cuidado informal en personas con insuficiencia crónica (Velázquez y Espín, 2014), personas mayores (López, 2013) siendo alejadas de los diagnósticos tomados para la investigación aquí presentada, no obstante, se encontraron estudios realizados con cuidadores de personas con enfermedad mental grave, en su mayoría desarrolladas por enfermería o medicina (Izquierdo et al., 2016) o desde la psicología clínica (Palacios y Jiménez, 2008) y 
han sido de tipo cuantitativo. Otras investigaciones han sido de tipo documental (Porcar et al., 2015) o son caracterizaciones sobre sus condiciones sociales y económicas (Gómez et al., 2016) pero no dan cuenta de la percepción que tiene el cuidador. Por todo ello se hace evidente la necesidad de realizar una investigación de interés interpretativo en la que se permita un acercamiento de la realidad social del cuidador informal desde su cotidianidad y percepción de síndrome de carga.

\section{Referente teórico}

Para el desarrollo del referente teórico de la investigación se recopilaron algunas perspectivas que han abordado y teorizado el cuidado, así pues, Kristen Swanson (2006) lo define como una relación interpersonal con un ser preciado por el que siente un compromiso y responsabilidad en proteger por medio de cinco procesos: un primero que corresponde a conocimientos; los cuales para esta investigación se relacionan con las capacidades que de manera técnica o empírica ha adquirido el cuidador informal para dar respuesta a las necesidades de la persona cuidada; el segundo es estar con, el cual se entiende como las preocupaciones y compromisos individuales que orientan la labor de cuidado; el tercero, hacer por, en el que se hace alusión a las condiciones enfermera-cliente-organización; como cuarto se encuentra el proceso concerniente a posibilitar, que se relaciona con las acciones de los cuidados dados y, finalmente, mantener las creencias, el cual está relacionado con las consecuencias y resultados en la relación de cuidado.

De manera análoga, Urra et al. (2011) lo definen como "el trabajo profesional destinado a la satisfacción de necesidades humanas en el ámbito de la salud de un individuo, comunidad o población, como también integrativo y holístico, porque abarca los diferentes estratos de necesidades" (p.18).

Margaret Jean Watson (2006) expone una teoría impregnada de pensamientos postmodernos y en la que comprende una base moral y filosófica que contiene diez factores caritativos en el proceso de cuidar desde la enfermería, los cuales son desarrollados bajo supuestos y posteriormente son abordados por la misma autora como Caritas de Cuidados (2007) como una actualización a su teoría. A partir del desarrollo de estos diez factores, la autora explica y delimita con ellos el cuidado profesional y los alcances que el cuidador tiene desde el desarrollo de su labor y la interacción con la persona cuidada.

Desde otras perspectivas, el cuidado ha sido abordado como una actitud esencial más que un acto o una actitud (Heidegger, 1951) en este sentido, se comprende que el cuidado se encuentra a priori de todo ser humano (Heidegger, 1951), para Carol Gilligan (1982) el cuidado trasciende de una ética de la justicia expuesta por Kohlberg (1981) a una ética del cuidado en la cual emerge la responsabilidad sobre el otro concreto que no corresponde únicamente a la mujer. En este sentido, el cuidado comprende la búsqueda de bienestar de quien se cuida mediante el reconocimiento afectivo, siendo el camino que permite a las personas comprenderse sobre el otro por medio del principio de "atención amorosa al bienestar del otro a la luz de sus necesidades individuales" (Honneth, 2006: 111), de allí que emerja una relación de cuidado basada en los sentimientos en la que se brinda un cuidado incondicional sobre las necesidades del otro (Honneth, 1997) los cuales son reflejados en relaciones de familia, amistad o pareja (Aparicio, 2016). 
El cuidado se materializa en una relación de cuidado, que para el caso de esta investigación es el escenario familiar, el que se comprende como una estructura intersubjetiva (Honneth, 2006) en la que se presentan relaciones de cuidado entre sus miembros familiares para la búsqueda de su mayor autonomía. De igual manera, se presenta como el escenario de socialización (Berger y Luckmann, 1966) donde la familia debe garantizar a la persona con enfermedad mental su vinculación a escenarios sociales y familiares con el fin de mitigar su marginación; así, el cuidado familiar trasciende de las necesidades físicas y psicológicas de la persona atendida puesto que responde también a ámbitos culturales, lúdicos y sociales para garantizar un bienestar (Aparicio, 2016).

Ahora bien, el desarrollo conceptual del cuidador Floréz (citado por De los Reyes, 2001) lo plantea como toda persona que asiste a otra que presenta cualquier tipo de dificultad que le impide desarrollar actividades diarias o vincularse a espacios sociales de manera independiente. Dicha labor puede desarrollarse de manera formal e informal.

El cuidador informal desarrolla su labor de manera artesanal, con amor y dedicación ante la adversidad (De la Cuesta, 2004); en ocasiones, atraviesa tensiones que pueden llevar a sensaciones de cansancio y carga traducidos en un síndrome de burnout cuyos síntomas fueron estudiados desde 1974 por Freundenberger al encontrar en un grupo de personas la presencia de desgaste, cansancio por exceso de trabajo, fuerza o recursos (Gutiérrez Aceves et al., 2006: 306) siendo estos síntomas que los cuidadores informales pueden atravesar.

En este mismo sentido, Renato Zambrano Cruz (2007) plantea que el síndrome de burnout se manifiesta a partir de síntomas y factores como dificultades en la salud física, acumulación de estresores, cambios conductuales del paciente que se cuida, altos costes económicos para responder a sus necesidades y las de quien se cuida, limitación de actividades propias que generen satisfacción personal. De igual manera, Castón (2009) plantea que la sensación de carga puede llevar a trastornos del patrón del sueño, irritabilidad, altos niveles de ansiedad, reacción exagerada a las críticas, dificultad en las relaciones interpersonales, sentimientos de desesperanza, resentimiento hacía la persona que cuida, pensamientos de suicidio y abandono, pérdida de energía, aislamiento social y pérdida de interés, causando como desencadenante afectaciones a nivel físico, mental y emocional en los cuidadores y posibles maltratos en la persona cuidada.

El síndrome de burnout ha sido contemplado desde dos tipos de cargas, la objetiva y la subjetiva (Espín, 2013); en ese mismo sentido, Montorio Cerrato (1998) indica que "además de la diferenciación entre carga objetiva y subjetiva, otras dimensiones más específicas de la carga del cuidador con las que se ha trabajado son, por ejemplo, la carga económica, física, intrapersonal y social" (p. 232); siendo así, investigaciones adelantadas han articulado la carga económica y social con la carga objetiva o estructural y la carga subjetiva o individual con la carga física e interpersonal, desarrolladas de la siguiente manera:

- Carga estructural: es expuesta como carga objetiva (Espín, 2013) y está relacionada con la cantidad de tiempo o dinero invertido en la labor de cuidado así como los problemas comportamentales de la persona que se cuida; en concordancia con esta afirmación se cuenta con el planteamiento de Fernández Gonzáles (2006), quien refiere que dentro de esta carga están presentes acontecimientos y actividades desarrolladas por el cuidador que limitan su vinculación a espacios laborales para tener un ingreso económico, lo que trae como problema colateral el incremento de gastos que no pueden ser sustentados por el cuidador y el desarrollo de una carga económica. Otro tipo de dificultad que puede presentarse es 
la limitación en el acceso a espacios sociales y familiares en los que pueda tener momentos de esparcimiento y ocio, llevando a que se produzca una carga de tipo social.

- Carga individual: también conocida como carga subjetiva, es entendida como la "percepción del cuidador de la repercusión emocional de las demandas o de los problemas relacionados con el acto de cuidar" (Espín, 2013: 3). Otros postulados indican que este tipo de carga connota los sentimientos que el cuidador tiene inmersos en su labor de cuidado y el desencadenante de ello en su salud física (Fernández, 2006). En este sentido, se visualiza que de este tipo de carga se desprenden las cargas de tipo emocional, psicológico (Montorio, 1998) y físicas (De la Cuesta, 2004).

En conclusión, las repercusiones de la carga en los cuidadores informales implican problemas de salud mental y física, tiempo libre, recreación o problemas económicos, entre otros. "Es así que la sobrecarga en el cuidador conlleva a consecuencias las cuales vivencia la persona en condición de discapacidad es relacionando estas en la claudicación o el abandono el cuidado, la institucionalización e incluso con malos tratos y abusos hacia las personas dependientes" (Carretero, 2006: 91). Lo anterior genera problemas tanto en el cuidador como en la persona con discapacidad a causa de los eventos estresantes gestados por la labor de cuidado, así como en el desarrollo de dependencias en la relación de cuidado (Fine y Glendinning, 2005).

\section{Marco metodológico}

\subsection{Enfoque y método de investigación}

La investigación partió de una perspectiva hermenéutica (Gadamer, 1977) a partir de la estratégica metodológica de análisis de conversación y de contenido (Sandoval, 1996) dado el interés de interpretar las percepciones de los cuidadores informales en relación a la carga en la labor percibida en su ejercicio de cuidado sin fines de alcanzar generalizaciones. El proceso de recolección de información tuvo una duración de dos meses; inicialmente se aplicaron entrevistas no estructuradas de manera individual en aras de obtener información desde una narración libre (Bonilla y Rodríguez, 1997) y explorar ampliamente la vida del cuidador informal y el ejercicio de cuidado en relación al tiempo, gastos, experiencias, percepciones y sentimientos que desde allí emergen.

Asimismo, en aras de ampliar la información obtenida por medio de las entrevistas individuales, se hace uso de la técnica de grupo focal (Bonilla y Rodríguez, 1997) en las instalaciones de la institución en la que realizó la investigación. Se desarrollaron preguntas alrededor de la labor de cuidado orientadas por las investigadoras como moderadoras en el abordaje del tema (López, 2010) para conocer las divergencias y convergencias de la concepción y percepción de la labor de cuidado desde las inferencias de los participantes.

\subsection{Participantes}

La configuración de la muestra se delimitó bajo la característica de ser cuidadores informales de personas con enfermedad mental toda vez que hacían parte del $60 \%$ de la población atendida, según la base de 
datos de la institución en la que se llevó a cabo la investigación. Se contó con la participación voluntaria de 12 cuidadores informales de personas con trastorno afectivo bipolar y esquizofrenia; cinco (5) cuidadores participaron en entrevista no estructurada y siete (7) en grupo focal.

Los participantes conforman un grupo con algunas características homogéneas como son: que la persona cuidada se encontrara adscrita a los servicios brindados por la institución en la que se desarrolló la investigación; residir en la localidad ${ }^{1}$ en la que se encuentra ubicada; y, finalmente, encontrarse en estrato socioeconómico 1, 2 y 3 de los 6 estratos que se cuentan en la ciudad de Bogotá2.

Los cuidadores en su mayoría fueron madres de las personas con discapacidad o cónyuges hombres a quienes se mantuvo en anonimato y asignado un número para la presentación de resultados. Cabe resaltar que no se tuvo oportunidad de abordar cónyuges mujeres. Aunque se abrió la convocatoria a todos los cuidadores informales, muchos refirieron no poder participar por disponibilidad de tiempo.

Por otro lado, no se contempló un rango de edad que delimitara su participación, aunque se evidencia por medio de caracterización facilitada por la institución, que las edades oscilaron entre los 18 a 68 años. En cuanto a su nivel educativo muchos presentan educación sin culminar, en algunos casos debido a embarazo en adolescencia o desplazamiento del sector rural al urbano, donde se vincularon a trabajos informales.

Tabla 1: Relación de cuidadores participantes

\begin{tabular}{|c|c|c|}
\hline Cuidador & Sexo & Parentesco \\
\hline \multicolumn{3}{|c|}{ Grupo Focal } \\
\hline Cuidador 1 & Hombre & Esposo \\
\hline Cuidador 2 & Hombre & Esposo \\
\hline Cuidador 3 & Mujer & Madre \\
\hline Cuidador 4 & Mujer & Madre \\
\hline Cuidador 5 & Mujer & Madre \\
\hline Cuidador 6 & Mujer & Madre \\
\hline Cuidador 7 & Mujer & Madre \\
\hline \multicolumn{3}{|c|}{ Entrevistas individuales } \\
\hline Cuidador 8 & Mujer & Madre \\
\hline Cuidador 9 & Mujer & Madre \\
\hline Cuidador 10 & Mujer & Madre \\
\hline Cuidador 11 & Mujer & Madre \\
\hline Cuidador 12 & Hombre & Esposo \\
\hline
\end{tabular}

Fuente: elaboración propia.

1. División administrativa de un territorio.

2. El estrato socioeconómico en Bogotá se comprende como una escala del uno al seis; dichos números corresponden a la clasificación que se da a la zona y los inmuebles en los que una persona reside para establecer de manera diferencial el cobro de cada servicio público, lo que conlleva a que un estrato 1 pague un costo mejor a un estrato 6, además, esta característica se abordó dado que los cuidadores adscritos a la institución donde se llevó a cabo la investigación se encontraban en los 3 primeros niveles de estratificación, siendo considerados los niveles más bajos dadas las dificultades sociales y económicas para responder a sus necesidades básicas, y por ende, los que pueden acceder a ayudas estatales. 


\subsection{Procedimiento}

El desarrollo de la investigación presentada contempló cuatro fases:

- Fase uno: diseño de instrumento y aplicación para recolección de información: tras la presentación de la investigación y firma de consentimiento informado por cada participante, se lleva a cabo la aplicación de entrevistas no estructuradas individuales de una duración de 30 minutos y aplicadas en el domicilio del cuidador; se abordaron temas alrededor las necesidades que desde emergen desde el cuidado y la percepción que tiene sobre su práctica, la sensación de carga. De igual manera se aplicó grupo focal en el que se abordaron preguntas alrededor del tema. Las entrevistas fueron grabadas para posteriormente ser transcritas, analizadas e interpretadas.

- Fase dos: transcripción original de la información recolectada y análisis (Andréu, 2013). Al lograr una saturación de información alrededor de las categorías deductivas por medio de las técnicas implementadas, se organizó la información en categorías deductivas.

- Fase tres: comprobar la fiabilidad del sistema de codificación-categorización. Se realizó un análisis de los hallazgos en el que se establecen las relaciones entre categorías y subcategorías y se hace registro de categorías inductivas que emergieron en la investigación, teniendo en cuenta las reglas de codificación.

- Fase cuatro: inferencias. Se finaliza el proceso de categorización por medio de la elaboración de taxonomías para establecer un orden entre causas, consecuencias y características del síndrome de carga del cuidador, alrededor de las cargas individuales y estructurales.

\section{Resultados y discusión}

Dentro de este apartado se presentan los resultados obtenidos producto del análisis realizado a partir de las categorías abordadas alrededor de la carga estructural e individual para interpretar la percepción del cuidador informal sobre su labor en relación al síndrome de carga:

\subsection{Carga estructural}

\section{- Carga económica}

A partir de las manifestaciones de algunos participantes, se comprende que la cantidad de tiempo invertido en el cuidado no solo es destinado para la atención de la persona cuidada, pues en muchas ocasiones deben asumir el cuidado de otros miembros de su hogar lo que limita su posibilidad de vincularse a un trabajo formal fuera del hogar (Fernández, 2006):

"Yo estoy de médica, enfermera, ama de casa, mamá, todo me toca (...) para darle de comer, bañarlo, todo..., no puedo trabajar, me toca solamente en la casa". (Cuidador $\mathbf{n}^{\circ} \mathbf{7}$ ) 
"Lo más difícil digamos... que uno a veces se le presentan oportunidades y no puede aprovecharlas pues...como digamos, yo por lo menos digo, yo no puedo salir a trabajar...". (Cuidador nº 9)

Conforme a lo expuesto se evidencia que el cuidado connota múltiples labores que reducen las oportunidades de asumir un empleo; sumado a ello, los cuidadores perciben una carga económica al no poder responder a sus necesidades como tampoco contar con un apoyo en este aspecto por parte de su familia:

"La familia así tenga la plata no le ayuda a uno". (Cuidador $\mathbf{n}^{\circ}$ 5)

"Mi mamá me la entregó cuando ella tenía dieciocho años, de ahí para acá pues yo he sido la que he vivido con ella y...y la he mantenido pero ha sido muy difícil (...) yo no tengo apoyo de nadie...económicamente". (Cuidador $\mathbf{n}^{\circ}$ 9)

Las situaciones manifestadas pueden llevar a que, en algunos casos, se presenten no solamente desequilibrios económicos que ponen en riesgo la estabilidad en este ámbito, sino que los efectos derivados de la crisis económica se trasladan a los ámbitos personales-emocionales. Por otro lado, se logra evidenciar que su labor de cuidado es delegada por miembros de la familia y es concebida como una práctica que puede generar carga de tipo económico cuando el cuidador no recibe apoyo por parte de sus familiares en la labor o gastos económicos del cuidado. La afirmación aquí expuesta se confirma al ser manifestada por los cuidadores la existencia de necesidades económicas que no pueden ser asumidas por ellos debido a su alto coste:

"La familia al principio le dice a uno, sí, tranquilo, pero, primero que todo a uno lo juzgan, que de pronto uno ha sido malo, que se ha (...) que ese es el castigo que dios le mandó a uno". (Cuidador nº 8)

"La situación económica sí es muy difícil porque con ellos gasta uno mucha plata”. (Cuidador n 5)

Por otro lado, se evidencia que la carga económica percibida en el cuidador puede generar cargas de tipo emocional (Gutiérrez Aceves et al., 2006) siendo un riesgo para la relación de cuidado teniendo en cuenta que el cuidador gesta también una percepción hacia la persona cuidada desde la relación intersubjetiva:

"Eso a mí me desespera... A mí lo que me preocupa, es él, atrae mucho más...más no aportan nada, nada". (Cuidador $\left.n^{\circ} 3\right)$

Por otro lado, para los cuidadores informales el apoyo institucional y especialmente estatal no ha definido unas líneas de acción claras para su atención, considerando que dentro de estas podrían ser vinculados laboralmente como estrategia en miras a mitigar sus necesidades económicas:

"Que de pronto el gobierno nos diera la oportunidad o sea en alguna entidad, yo por ejemplo, yo podría trabajar, y de pronto en alguna entidad donde me den un trabajo a mí de noche". (Cuidador $\mathrm{n}^{\circ}$ 12)

De igual manera, los cuidadores informales consideran factible la incorporación de personas con enfermedad mental en trabajos formales que puedan desarrollar según sus capacidades y de allí mejorar la situación económica del hogar desde la inclusión social de la persona cuidada en espacios externos; es así como se evidencia que para el cuidador su carga percibida puede ser mitigada a partir de procesos de vinculación social para la persona cuidada, en los que la familia no sea el único responsable de garantizarlo, lo cual se acerca a las limitaciones expuestas por Etxeberría (2005): 
"Ellos (refiriéndose al Estado) pueden darnos un apoyo económico, poder fundar como una institución para niños especiales, para que puedan aprender algo y se puedan desempeñar por sí mismos”. (Cuidador nº

Los cuidadores refieren percibir que la activación de redes institucionales presenta dificultades por los criterios de ingreso para algunos servicios estatales debido a que no cumplen con los requisitos solicitados; por otro lado, perciben que su ejercicio de cuidado es demandado pero no es apoyado por otros actores o entidades dada la complejidad del diagnóstico que presenta la persona cuidada; dichas situaciones percibidas conllevan que exista una red de apoyo institucional débil:

"Hemos buscado apoyo (en referencia a la atención estatal); como en el carnet tenemos un puntaje muy alto, que me salió, entonces he sido rechazada". (Cuidador $n^{\circ} 11$ )

"Yo estuve buscando apoyo por parte de la alcaldía de Bogotá, pero allá necesitan niños que caminen, que se defiendan solos y que lo que el niño necesitaba era atención personalizada". (Cuidador $n^{\circ}$ 5)

Las situaciones expuestas conllevan que el cuidador informal esté en riesgo de sentir carga de tipo emocional debido a las gestiones que realiza pero que son denegadas, no dando respuesta a sus necesidades (Gutiérrez Aceves et al., 2006), lo cual conlleva a percibir afectaciones en su bienestar.

Otro aspecto que los cuidadores consideran un factor que genera carga económica es el tiempo destinado a la gestión para la atención en salud de las personas cuidadas, puesto que muchos de estos procesos no contemplan el apoyo de un profesional que les oriente el proceso en aras de optimizar tiempo y reducir costes de movilización, por lo que se genera desgaste y cansancio debido al exceso de recursos y fuerzas que debe usar (Gutiérrez Aceves et al., 2006).

"Como es que uno va a llegar un hospital y le van a decir que...y no le van a decir a uno bueno usted tiene que hacer esto, y esto, y esto...nada no hay una persona que lo oriente a uno". (Cuidador $\mathbf{n}^{\circ}$ 9)

Lo anterior evidencia que para los cuidadores informales existe una figura estatal en la que se encuentran diferentes proyectos de ley, sin embargo, consideran que no se está brindando por parte de esta redes de apoyo que permitan solventar sus necesidades y el reconocimiento de sus derechos para el alcance del bienestar de esta población y las personas que cuidan. No obstante, queda patente que el cuidador busca de manera estratégica desarrollar su labor de cuidado ante las adversidades (De la Cuesta, 2004) en aras de tener una remuneración de tipo económico al desarrollar trabajos independientes, por lo tanto, su rol de cuidador entra en interacción con otros roles como el de trabajador informal:

"Pues yo hago por ahí cositas; precisamente estaba allá arriba haciendo cositas de carpintería porque, pues no me puedo comprometer con cosas grandes porque como me toca llevarla al médico, me toca estar pendiente de ella, entonces no, no puedo trabajar en una empresa, ni nada". (Cuidador $\mathbf{n}^{\circ}$ 12)

"Trabajo como independiente, porque hay que dedicarle tiempo y es como si fuera un niño...". (Cuidador $\left.\mathbf{n}^{\circ} \mathbf{7}\right)$

La informalidad no solo la tienen en su figura de cuidadores sino que también tienen que ejercer la informalidad laboral, sometiéndose a la inestabilidad que esta genera y repercutiendo directamente en el desenvolvimiento adecuado de sus vidas; de ahí que se manifieste el sentir de carga en este aspecto y se deriven los efectos sociales de la misma. 
De igual forma, la suma de los diversos gastos que tienen que afrontar a diario (pañales, medicamentos, alimentación, transporte) más las demandas en tiempo del cuidado, la falta de apoyo estatal sobre la mediación de bonificaciones que de alguna manera amortigüen la carga económica hacen que el cuidador informal se vea marginado y en situación de pobreza. Así pues, lo mencionado refleja lo expuesto por Esping (2000) alrededor de la mercantilización del trabajo; en este caso es evidente que la labor del cuidador informal no se reconoce monetariamente, lo que conlleva que el cuidador deba garantizar un cuidado óptimo para la persona que requiere de su atención pese a que no cuenta con los recursos suficientes para responder en su totalidad en la gestión de los elementos necesarios para satisfacer las necesidades de la persona cuidada, gestándose una percepción de carga económica en el cuidado.

\section{- Carga social}

La importancia del ser social implica directamente la activación de redes en todos los campos (institucionales, familiares, relaciones de amistad), de igual manera, el ser humano desde su nacimiento requiere de cuidados (Heidegger, 1951), de ahí que la familia sea identificada como una fuente de protección hacia el bienestar de sus miembros (Esping, 2000), en el que el cuidador debería ser cuidado desde la concepción del reconocimiento afectivo expuesto por Honneth (1997), donde la cooperación entre familiares para el ejercicio del cuidado se vería materializada; sin embargo, algunos cuidadores informales frente a su cotidianidad perciben la falta de apoyo y aislamiento por parte de sus familiares $y / o$ amigos dado que el cuidado que ejercen es permanente e implica un compromiso que no todos desean asumir, esto hace que perciban ausencia de bienestar y estabilidad emocional siendo esto un riesgo para su salud mental:

"Con mi familia sí, les da pena con mi bebe (...) pues nunca he tenido el apoyo de mi mamá, alguien que le dé apoyo, una palabra de aliento, apoyo cuando uno lo necesita". (Cuidador $\left.n^{\circ} 10\right)$

Existen otros casos en los que los cuidadores informales manifiestan tener una red familiar que les apoya en su labor de cuidado, de ahí que perciban que también son miembros cuidados desde el apoyo que brindan los otros, lo cual amortigua la percepción de carga en el cuidador:

"Por mi mamá, que es la que más moral me ha dado en estos tiempos de esta enfermedad que le dio a ella". (Cuidador $n^{\circ}$ 12)

La dedicación de cuidado informal es permanente, lo que implica que la participación del cuidador en espacios sociales y familiares se limite o afecte en su totalidad generando sensaciones de carga debido al aislamiento social y familiar percibido por el cuidador:

"Se le dañan las amistades, la familia se le aísla a uno, a mí me pasó". (Cuidador n 3 )
"La vida social a uno se le daña porque no es por nada pero es como si fuera una carga para uno...". (Cuidador n ${ }^{1)}$

También se evidencian problemas que recaen sobre aspectos referidos a las relaciones con otros integrantes de la familia cuando el cuidador debe cuidar el bienestar de otros miembros; esta situación se acerca a los planteamientos expuestos por Velasco y Sinibaldi (2001) alrededor de los desequilibrios que afrontan las familias al presentarse una enfermedad dentro del sistema familiar: 
"Los deberes del cuidador, pues me han...me han dado ciertos problemas, (...) como me puse a cuidar a la mamá pues descuidé a la hija, (...) actualmente la tengo interna por consumo de alucinógenos". (Cuidador $\mathbf{n}^{\circ}$ 1)

Pero, más allá de poder identificar la ruptura de este vínculo o apoyo, se debe analizar la construcción generalizada sobre la carga; no solamente el cuidador la manifiesta sino que el desapego y designación y responsabilidades del cuidado a un individuo de la familia también enseña que la sociedad ve la discapacidad como una limitación y cadena, por lo que la mayoría opta por abortar esta labor, lo que genera en el cuidador mayor percepción de carga emocional al verse señalado y marginado por su familia:

"La familia al principio le dice a uno, sí, tranquilo, pero, primero que todo a uno lo juzgan, que de pronto uno ha sido malo, que se ha (...) que ese es el castigo que dios le mandó a uno". (Cuidador n 8)

Lo manifestado como ausencia de apoyo familiar para el cuidador lleva a que se gesten emociones de tristeza, odio, sensación de soledad, siendo estos factores que inciden en la estabilidad de la salud mental del cuidador conforme han sido descritas por Castón (2009). De igual manera, la relación de cuidado se tensiona en la medida que la persona cuidadora percibe cansancio por la situación familiar, social y económica afrontada y, en ocasiones, genera señalamientos sobre la persona cuidada.

Existen otros casos en los que el cuidador informal y su familia deben cambiar su cotidianidad en cuanto se asume su rol; las atenciones y la gestión de procesos de salud que no son brindados en los territorios donde residen les implican migrar de sus zonas de origen, lo que demuestra que su labor se desarrolla desde la adversidad (De la Cuesta, 2004):

"Es una situación difícil, mi vida dio un giro de 90 grados. Y o sea mi vida cambió, yo tenía mi hogar, mi familia, mi trabajo fijo, y pues nos vinimos acá para el tratamiento de él, la rehabilitación". (Cuidador nº 7 )

Por otro lado, cuando los diagnósticos se presentan en personas adultas que están casadas o en unión libre, en muchas ocasiones son los cónyuges los que asumen el cuidado de la persona; este cambio de rol y la situación de salud atendida lleva aparejada la transformación de la relación afectiva a una relación de cuidado asistencial orientada al bienestar de la salud, donde la persona cuidada ya no es identificada como la pareja del cuidador, sino que es percibida como una paciente que requiere de cuidados:

"En cuanto a la relación de pareja, no, eso sí se pierde, pues yo a ella la quiero mucho, pero...pues ella ya en pocas palabras ella ya se perdió, así como mujer, que ella ya a uno, ella así enferma uno no va a poder siquiera sacarla a un parque, no va a poder llevarla a un almuerzo". (Cuidador $\mathbf{n}^{\circ}$ 12)

De igual manera, se evidencia que el cuidador cuando es el cónyuge asume la labor como una práctica que es únicamente de su responsabilidad, al concebir que los hijos fruto de dicha unión deben continuar con su vida; lo mencionado refleja que el cuidado del cuidador trasciende a ser un ejercicio brindado a todos los miembros familiares y es concebido como un acto de amor (De la Cuesta, 2004) aunque perciban que dentro de ella se gestan sensaciones de carga:

"Cuidador de mi esposa desde hace el espacio de 12 años, ella depende, pues de la familia, prácticamente de mí, pues porque-tenemos 7 hijos, solo 7 hijos-pero todos tienen su hogar por lo tanto ellos no pueden asumir esa carga (...) es una carga, pero yo la verdad ya vivo como acostumbrao, resignao". (Cuidador $\mathbf{n}^{\circ}$ 2) 
A esto se suma la discriminación social que sobre la enfermedad se tiene que es percibida por el cuidador, representada en la infraestructura de la ciudad, el transporte público, las burlas, etc., generando en los cuidadores miedo al rechazo y por consiguiente haciendo que las personas consideren más el tiempo de quedarse en casa, culminar con las amistades y salidas, entre otros aspectos; estas condiciones derivan en que el cuidador se sienta incapaz de salir, tenga dificultades para trabajar fuera de casa, por lo tanto, se reducen sus procesos de socialización secundaria (Berger y Luckmann, 1966) e implica afectaciones emocionales que inciden directamente en su salud mental del cuidador.

El rechazo, la discriminación y la exclusión familiar en ocasiones puede llevar al surgimiento de una relación de co-dependencia desarrollada en el ámbito del cuidado. La existencia de este hecho señala otro foco dentro del tema de cuidadores, trazando un nivel de importancia para nutrir el ámbito investigativo en relación con la perspectiva de género, teniendo en cuenta que prevalece el ejercicio de cuidado informal en la mujer, especialmente en progenitoras.

Se evidencia de esta manera que las repercusiones que sobre el cuidador acontecen influencian también el tipo de relación que tienen con la persona a la que cuidan, así, para ahondar un poco más sobre este hecho, se ha de analizar los aspectos que componen la carga subjetiva, siendo estos los interpersonales y los físicos.

\subsection{Carga individual}

\section{- Carga intrapersonal}

Para los cuidadores desempeñar su rol implica conocimiento y dedicación y compromiso (De la Cuesta, 2004), caridad y utilidad sentida por sus seres queridos; así pues, su labor se realiza por medio de ofrecimientos de amor a la persona cuidada, en el que el desvelo y la atención están presentes (Boff, 2002). Por otro lado, el cuidador manifiesta recibir amor por parte de la persona atendida, lo cual hace que exista una interacción recíproca como símbolo de unión y de dedicación que en la mayoría de los casos es manifestada por la madre quien desarrolla una práctica de cuidado incondicional (Honneth, 1997):

\footnotetext{
"Tener toda la energía y todo el amor a los hijos, porque yo creo que en esta labor nadie como ser una mamá que se dedica a ellos". (Cuidador $\left.\mathbf{n}^{\circ} \mathbf{8}\right)$

"Me ha dado el amor que nunca tuve, me da el amor, lo siento tan mío, es tan tierno, él me da todo lo que necesito, es lo mejor que le pasa a una mamá, una mamá debería apegarse a ellos". (Cuidador $\mathbf{n}^{\circ}$ 10)
}

Hay un tópico generalizado alrededor del cuidado en los participantes y es el sentimiento de amor; todos los cuidadores lo manifiestan como la entrega total que deben tener con la persona, lo cual se acerca a afirmaciones como las de Boff (2002), como también a las manifestaciones afectivas desarrolladas por Honneth (2006), sin embargo, la construcción de amor se ve relacionada en la mayoría de los casos con el sacrificio percibido por el cuidado:

"Pues muchas veces las mamás sí lo sentimos como una carga, porque habemos mujeres que si quisiéramos tirar la toalla (...) todo eso le, le, le, le daña a uno la vida, es una carga muy tenaz...". (Cuidador $\mathbf{n}^{\circ}$ 12) 
Así, se pueden explicar los sentimientos que de allí derivan como la resignación y la culpabilidad mezclados con sentimientos de satisfacción por la labor realizada, conformidad, preocupación e impotencia, cansancio, sensación de ser manipulados por parte de la persona a la que cuidan e incluso llegando a relaciones conflictivas. Aquí se resalta la ambigüedad que sobre la entrega se tiene:

"A veces como... se siente un desespero muy tremendo porque a veces se siente...impotente de poder hacer algo por esta persona más que todo en los momentos de crisis...manejar una persona, una esquizofrénica es tenaz... el calmar a la persona es muy difícil...". (Cuidador $n^{\circ}$ 9)

Ahora bien, la relación de cuidado presentada refleja un acercamiento a los planteamientos expuestos por Bateson et al. (1956) en la medida en que se desarrollan dobles mensajes dentro de la interacción entre el cuidador y la persona cuidada; el cuidador percibe que su cuidado es requerido y se lleva a cabo con amor pero pueden producirse actos hostiles por parte de la persona cuidada como respuesta ante sus cuidados. De este modo, en la relación de cuidado se presenta una interacción intersubjetiva (Honneth, 1997) que presenta tensiones cuando los actores de la relación de cuidado no se perciben como sujetos reconocidos por el otro concreto al momento de recibir un doble mensaje:

"Él tiene 34 años, pero eso ha sido muy difícil, muy difícil (prosigue el llanto) a él toca hacerle la comida y servírsela y más encima... (pausa por llanto), a uno lo trata mal,...., (...) a ratos parece que se aprovecha”. (Cuidador $\mathbf{n}^{\circ}$ 11)

Estas situaciones generan carga emocional a partir de los sentimientos que emergen desde el ejercicio de cuidado que se lleva a cabo (Espín, 2013) y puede repercutir en sensaciones de frustración debido a su confusión; bajo esta situación, algunos participantes establecen como apoyo emocional su acercamiento a una deidad:

"Me dan unas ganas de chillar, me da desespero que no puedo hacer algo por él, yo quisiera que ya no le diera esa epilepsia (...) he llegado al punto de que a veces digo: uy, Dios mío, yo qué camino cojo, para dónde agarro (...) mi único camino que yo he visto, y esto es pedirle a Dios que me dé la paciencia”. (Cuidador n 9)

Sin embargo, existen casos particulares que no manifiestan carga en este aspecto, y son más congruentes con el significado del amor, manifestando felicidad con el hecho de cuidar, "bendiciones", en palabras textuales sobre lo que la persona le significa al cuidador $\mathrm{y}$, por tanto, amortiguando directamente la carga que pueden manifestar en otra área, por ejemplo, la económica:

"Pues para mí la experiencia no ha sido tan difícil porque él no ha sido así tan enfermito. Y pues no, así como que me haya parecido carga... carga no. Y yo no siento que esté pagando nada porque gracias a Dios no siento que deba nada". (Cuidador $\mathbf{n}^{\circ}$ 5)

"Lo amo demasiado, mi familia también (...) yo le digo a todas las mamás que deberían tener mucho amor con estos niños, que ellos entienden más que cualquier niño normal, ellos son normales, yo digo que no, hay que hacerlo con amor". (Cuidador $\mathrm{n}^{\circ}$ 10)

Se identifica que la conclusión a la que llegan todos los cuidadores, pese a manifestar la carga en los aspectos nombrados, es el amor profundo que se ha de tener por ese ser al que cuidan y, de nuevo, el carácter maternal recoge su lugar en las afirmaciones: 


\footnotetext{
"Tener toda la energía y todo el amor a los hijos, porque yo creo que en esta labor nadie como ser una mamá que se dedica a ellos". (Cuidador $\left.\mathbf{n}^{\circ} \mathbf{8}\right)$

"Me ha dado el amor que nunca tuve, me da el amor, lo siento tan mío, es tan tierno, él me da todo lo que necesito, es lo mejor que le pasa a una mamá, una mamá debería apegarse a ellos". (Cuidador $n^{\circ} 10$ )
}

Este hecho se ve directamente relacionado con la temporalidad donde apareció la enfermedad de la persona a la que cuida; así, las personas que cuidan a aquellos que nacieron con la enfermedad conciben de una manera más aceptable el cuidado, mientras que los cuidadores cuyos parientes adquirieron la enfermedad en un determinado punto de la vida diferente del nacimiento tienden a asimilarlo de una manera negativa:

\footnotetext{
"Hoy no le interesa si es de día o es de noche, nada, él no...él no se acomide a nada!, y de ahí pa acá sufriendo todo, imagínese sumercé es duro, es duro pa uno, que uno no esperaba eso de él”. (Cuidador n 11)

"El neurólogo me dijo: "su niño le va a quedar ciego, un niño especial, o no va a caminar" y yo me puse y lloré y ya después yo misma me puse a pensar: es que los médicos no son Dios, si así me queda mi bebé no me importa, le dije a Dios, no me importa cómo me quede mi bebe, lo acepto, lo voy a querer y lo voy a cuidar hasta donde yo pueda". (Cuidador $\mathbf{n}^{\circ}$ 10)
}

Con lo anterior, se tiene en cuenta que la construcción cultural está directamente relacionada con el modo en el que se ha concebido históricamente la enfermedad y, por tanto, el cuidado; gran parte de ello deriva en la concepción judeo-cristiana del sacrificio relacionado con el amor.

Por otro lado, el rol del cuidador se mantiene como un papel delegado a la mujer (Daly y Lewis, 2000) en el que emergen las percepciones de cargas debido a las múltiples tareas que debe desarrollar, las cuales no son reconocidas ni remuneradas por los demás miembros de la familia; esta situación se presenta debido a que el cuidado informal no es visto como un trabajo dentro de los escenarios formales (Tobío, 2012):

\footnotetext{
"Habemos mujeres que sí quisiéramos tirar la toalla, porque tenemos que cargar con el hijo, tenemos que cargar con el esposo, tenemos que lidiar con todas esas obligaciones del hogar como mamá y como mujer y a uno no le ven eso, ni el esposo ni nada". (Cuidador $\mathbf{n}^{\circ}$ 12)
}

El cuidado está generando sentimientos de represión sobre la labor, de ahí que se puedan explicar las relaciones conflictivas que algunos manifiestan con la persona a la que cuidan, y obteniendo de allí sentimientos de culpa frente a los enfrentamientos al ser conscientes de que es una persona con enfermedad, al recordar que su enfermedad no es congénita o no es un diagnóstico que haya sido presentado en su nacimiento.

"Es muy difícil lidiar con una personita de esas, donde él no aporta nada sino, nada! (...) él no se preocupa ni por el aseo personal de él, toca tar pendiente,(...) uno se estresa, más que todo, preocupación (...) me tocó limitarme de todo, aguantar y tenerle paciencia a él”. (Cuidador $\left.n^{\circ} 11\right)$

Lo aquí expuesto permite tener un acercamiento a los planteamientos de Bateson et al. (1956) alrededor del doble vínculo, en el que se presentan dos situaciones y mensajes contradictorios; por un lado, la pérdida de vida social y, por otro, el amor y la oportunidad de vida para la persona a quien se cuida, conllevado a la codependencia. Esta situación puede llevar a una dicotomía en la percepción de la relación del cuidado 
en el cuidador: por un lado, su constante contacto con la persona cuidada y su deseo de dejar de ejercer el cuidado para no sentir carga, lo que puede conllevar a sentimientos de rechazo hacia su familiar y, por el otro lado, su necesidad de seguir cuidando para preservar la vida de quien requiere su atención y la búsqueda de hacer sentir bienestar a la persona cuidada. De igual manera, este proceso de doble mensaje en la comunicación desde la relación de cuidado puede conllevar confusiones a partir de lo percibido por la persona que recibe el cuidado.

Los resultados obtenidos permiten un acercamiento a las afectaciones emocionales que atraviesan los cuidadores informales debido al estrés de su labor, por lo que es necesario desarrollar estrategias orientadas al cuidado de la vida del cuidador que respondan a sus necesidades de manera efectiva en pro de prevenir enfermedades mentales en este.

\section{- Carga física}

En relación al aspecto físico se evidencia que los cuidadores informales refieren presentar repercusiones en este ámbito debido al esfuerzo corporal que deben realizar o las situaciones de tensión en la relación de cuidado debido a las alteraciones comportamentales presentadas en las personas manifestadas en violencia física, esto conlleva a presenciar acumulación de estresores como es expuesto por Zambrano (2007):

"Ella me cogió y me mechoneó y yo estaba como delicada de salud; yo he tenido artritis, entonces yo ya no tengo fuerzas en mis manos ni nada...y ella pues me dominaba, me dominaba...". (Cuidador $\mathbf{n}^{\circ}$ 9)

"Lo que me afectó fue una rodilla por bajarla, y subir las escaleras me compré una faja y ya, logré recuperar la rodilla y pues ahorita ya como bajó de kilos ya se me ha facilitado más". (Cuidador $\mathbf{n}^{\circ}{ }^{12}$ )

Las afirmaciones expuestas anteriormente permiten conocer que la cotidianidad del cuidador presenta tensiones alrededor de sus prácticas de autocuidado; de igual manera, su labor conlleva presentar malestares que en muchas ocasiones no son atendidos por la ciencia médica sino por ellos mismos por medio de automedicación. Cabe resaltar también que los cuidadores cuando son personas mayores presentan mayor riesgo de presentar enfermedades múltiples relacionadas con su ciclo vital:

"Afortunadamente ella es delgadita, ella no pesa, el peso de ella son 40 o 36 kilos. Vivo en un segundo piso y pues yo la bajo alzada, la acomodo en la silla de ruedas. Ya somos unos ancianos... Yo tengo 68, mi esposa 62". (Cuidador $n^{\circ} 2$ )

Como fue desarrollado en la subcategoría de carga emocional, la auto-anulación de los cuidadores, explicada por la entrega total a la labor del cuidado, hace que poco se referencien las implicaciones en salud que tienen sus tareas diarias, por lo que no lo consideran un aspecto relevante, ya que, si se nombra este aspecto, los cuidadores tienden a describir detalladamente la enfermedad de la persona cuidada, pero si se hace referencia a ellos mismos les cuesta recordar incluso qué padecen.

Resaltan bastante los aspectos emocionales que se ven afectados, por ejemplo, el sentirse heridos por la discriminación social, pero lo hacen por lo que les hagan a sus familiares más que a ellos mismos. 
En este orden de ideas y bajo este panorama analítico, se logra reflejar que la obtención de resultados permitió conocer las percepciones que tiene el cuidador sobre su labor, frente al sentir de carga que expresa textualmente, debido a los gastos que debe hacer para el cuidado, el daño de las relaciones sociales y familiares, la limitación que siente al no poder desplazarse a otros lugares, y el desarrollo de su proyecto de vida a nivel laboral y académico, entre otros aspectos ya mencionados.

\section{Conclusiones}

El proceso de investigación presentado en este artículo permitió tener un acercamiento a la percepción que los cuidadores informales tienen sobre las repercusiones colaterales que produce el ejercicio de su labor y profundizar en aspectos de la vida del cuidador informal desde su cotidianidad.

Los relatos de los cuidadores informales participantes de la investigación dan a conocer que su labor se desarrolla desde el vínculo con su persona cuidada en el cual el amor es la piedra angular en su ejercicio de atención a las necesidades de ese otro a quien se cuida, lo que implica que su cuidado no esté limitado solo a acciones someras que respondan a necesidades físicas, sino que busca responder a las necesidades emocionales que presenta la persona cuidada. No obstante, las tensiones y las dificultades que a nivel comunicacional emergen conllevan la producción de contradicciones en la percepción y concepción de la relación de cuidado en el que se refleja un cansancio del cuidador manifestado en ocasiones en desdén o deseos de que su labor de cuidado culmine versus la sensación de que el cuidado es un ejercicio que es parte de su vida y que, por ende, le da sentido a la misma.

Se evidencia que la literatura internacional ha hallado que el cuidado es un ejercicio desarrollado principalmente por mujeres, lo cual es reflejado en este estudio, pues la mayoría de cuidadoras eran progenitoras, lo que lleva a reflexionar sobre la importancia de continuar abordando el papel del hombre dentro del cuidado al presentarse también cuidadores dentro de la investigación.

Por otro lado, para algunos cuidadores la labor del cuidado no siempre constituye una carga, debido a que el tipo de relaciones que se establecen con la persona a la que cuidan mitiga otras de tipo externo (aspectos económicos y otros). Existe una percepción explícita de carga en el cuidador debido a que, en muchos casos, es el único cuidador familiar y quien debe asumir las múltiples las responsabilidades del hogar. Dicha situación acarrea que este aspecto trascienda a su emocionalidad y sea un riesgo para su salud mental, lo que lleva a la necesidad de establecer acciones estratégicas que brinden atención al cuidador en aras de prevenir enfermedades como depresión o ansiedad a consecuencia de su labor.

La participación de los cuidadores dentro de la investigación permitió un acercamiento a su vida cotidiana, su sentir y procesos subjetivos que son gestados, donde se evidencia una percepción de carga estructural e individual; sin embargo, logran expresar con mayor facilidad sus necesidades económicas y su ausencia de apoyo familiar y/o estatal así como sus afectaciones emocionales pero no identifican en muchas ocasiones la carga física debido a la auto-anulación que emerge en su labor de cuidado, viéndose esto como una limi- 
tación en la investigación. Por otro lado, se identifica la dificultad de algunos cuidadores para movilizarse de su casa y participar en el grupo focal.

Ahora bien, la auto-anulación del cuidador es identificada desde los primeros contactos; al preguntar cosas básicas sobre su vida ellos se refieren constantemente a la persona cuidada. Al delimitar la población, se logró identificar que cada cuidador tiene necesidades específicas relacionadas con el tipo de enfermedad o discapacidad que cuida y sus características demográficas (sexo, edad, barrio de residencia, estrato social y otros) las cuales enmarcan sus particularidades que el cuidador debe solventar.

A nivel nacional se ha buscado desarrollar acciones que den respuesta a las necesidades de los cuidadores, sin embargo, aún se requiere generar estrategias que respondan a la prevención de cargas que son desencadenadas por la labor constante de cuidar, pues se han desarrollado actividades de capacitaciones al cuidador cuando estos ya cuentan con habilidades de cuidado adquiridas de manera empírica; además, se refleja que han sido acciones someras y en las que se relega el papel del cuidador dentro de la relación de cuidado.

A nivel investigativo resulta relevante dar continuidad a este tipo de estudios en aras de tener conocimiento de la situación vivencial del cuidador informal; continuar abordando la subjetividad de los cuidadores y la intersubjetividad desde la relación de cuidado desde líneas de investigación que aborden temáticas tales como cuidado y subjetividad, familia y cuidado, cuidado y salud mental, cuidado y bienestar social, entre otras que aporten a la construcción de conocimiento para la mejora de la calidad de vida de los cuidadores desde el reconocimiento de su voz y de sus derechos a partir del cuidado al cuidador.

Finalmente, a nivel nacional es importante la construcción de una política pública que garantice al cuidador un reconocimiento legal, monetario y social sobre su labor de cuidado y sobre sus necesidades como sujeto, en la cual, este tipo de resultados permite que se conozca la voz de los sujetos como autores de la construcción de su realidad. Los procesos de atención domiciliaria también son necesarios, pues prevendrían en el cuidador desgaste físico y económico cuando debe movilizarse con la persona cuidada; así como el reconocimiento del cuidador como un miembro activo del sistema familiar, que presenta necesidades de ocio como los demás. Igualmente, es importante que se reconozcan las habilidades de las personas con enfermedad mental que pueden ser fortalecidas en espacios externos a su hogar, siendo una estrategia que ayudaría al cuidado, tal y como es manifestado por los cuidadores participantes de la investigación. 


\section{Referencias bibliográficas}

Abellán, A. y Esparza, C. (2010): Solidaridad Familiar y dependencia entre las personas mayores (en línea). <http:// envejecimiento.csic.es/documentos/documentos/pm-solidaridad-familiar.pdf>, acceso 25 de noviembre de 2019.

Agencia de Información Laboral (2016): Retirado proyecto de ley que eliminaba estabilidad laboral reforzada para trabajadores discapacitados (en línea). <https://aceb.org.co/retirado-proyecto-de-ley-que-eliminaba-estabilidad-laboral-reforzada-para-trabajadores-discapacitados/>, acceso 19 de junio de 2019

Aguas, S. (1999): “Una protección social invisible”. Hechos y Derechos, 6: 27-37.

American Psychiatric Association (2014): DSM-5. Manual diagnóstico y estadístico de los trastornos mentales. Arlington: American Psychiatric Association.

Andréu, J. (2013): Las técnicas de análisis de contenido: una revisión actualizada. Sevilla: Fundación Centro de Estudios Andaluces.

Aparicio, M. (2016): "El cuidado en la teoría del reconocimiento de Honneth. Repercusiones en las personas con diversidad funcional”. Oxímora Revista Internacional de Ética y Política, 8: 36-52.

Aretio, A. (2010): "Una mirada social al estigma de la enfermedad mental”. Cuadernos de Trabajo Social, 23: 239300.

Bateson, G. et al. (1956): Hacia una teoría de la esquizofrenia (en línea). <http://carlosreynoso.com.ar/archivos/ bateson-esquizofrenia-ok2.pdf>, acceso 19 de junio de 2019.

Berger, P. L. y Luckmann, T. (1966): La construcción social de la realidad. Buenos Aires: Amorrortu.

Boff, L. (2002): El cuidado esencial: ética de lo humano, compasión por la tierra. Madrid: Trotta.

Bonilla, E y Rodríguez, P. (1997): La investigación en Ciencias Sociales. Más allá del dilema de los métodos. Bogotá: Norma.

Carretero, S. et al. (2006): La sobrecarga de las cuidadoras de personas dependientes, análisis y propuestas de intervención psicosocial. Valencia: Tirant lo Blanch.

Castón, P. (2009): Cuidadores de personas mayores con discapacidad y unidades de estancia diurna en Andalucía. Sevilla: Fundación Centros de Estudios Andaluces.

Castro, R. et al. (2009): Necesidades de cuidado que tienen los cuidadores de personas en situación de discapacidad, por parte de su familia en la localidad de Fontibón. Bogotá: Pontificia Universidad Javeriana, Facultad de enfermería.

Congreso de Colombia (2014): Proyecto de Ley 62 de 2014 (en línea). <https://vlex.com.co/vid/proyecto-leysenado-525597126>, acceso 10 de mayo de 2018.

Congreso de Colombia (2010): Ley 1392 de 2010 (en línea). <https://www.minsalud.gov.co/sites/rid/Lists/BibliotecaDigital/RIDE/DE/DIJ/ley-1392-de-2010.pdf>, acceso 11 de agosto de 2017.

Congreso de Colombia (2009): Proyecto de Ley 33 de 2009 (en línea). <https://vlex.com.co/vid/proyecto-leysenado-451467698>, acceso 13 de marzo de 2013. 
Crespo, M y López, J. (2006): El apoyo a los cuidadores de familiares mayores dependientes en el hogar: desarrollo del programa "Cómo mantener su bienestar". Madrid: Instituto de Mayores y Servicios Sociales (IMSERSO).

Daly, M. y Lewis, J. (2000): "The concept of social care and the analysis of contemporary welfare states". British Journal of Sociology. 51 (2): 281-298.

De la Cuesta, C. (2004): Cuidado artesanal: la invención ante la adversidad. Antioquía, Colombia: Universidad de Antioquía.

De los Reyes, M. (2001): Construyendo el concepto de cuidador de ancianos (en línea). <https://gerincon.files. wordpress.com/2010/05/construyendo_el_concepto_de_cuidador.pdf>, acceso 12 de abril de 2013.

Espín, A. (2013): Factores de riesgo de carga en cuidadores informales de adultos mayores con demencia. Infomed, Red de salud de Cuba. (En línea): <http://scielo.sld.cu/scielo.php?script=sci_arttext\&pid=S0864$34662012000300006>$ acceso 10 de enero de 2018.

Espín, A. (2000): Fundamentos sociales de las economías posindustriales. Barcelona: Ariel.

Etxeberría, X. (2005): Aproximación ética a la discapacidad. Bilbao: Universidad de Deusto.

Fernández, C. (2006): Percepción de carga subjetiva en cuidadores de mayores dependientes. Proyecto de intervención (en línea). <https://www2.uned.es/intervencion-inclusion/documentos/Proyectos\%20realizados/ Carolina\%20Fernandez\%20Gonzalez.pdf>, acceso 12 de febrero de 2018.

Fine, M. y Glendinning, C. (2005): "Dependence, independence or inter-dependence? Revisiting the concepts of “care' and 'dependency'”. Ageing \& Society, 25: 601-621.

Gadamer, H. (1977): Verdad y método. Salamanca: Sígueme.

Gilligan, C. (1982): La moral y la teoría: psicología del desarrollo femenino. México D.F.: Fondo de Cultura Económica.

Gómez, A. et al. (2016): "Caracterización y condiciones de los cuidadores de personas con discapacidad severa en Bogotá". Revista Salud Pública, 18 (3): 367-378.

Gutiérrez Aceves, G. A. et al. (2006): "Síndrome de Burnout”. Medigraphic, 11 (4): 305-309.

Heidegger, M. (1951): El Ser y el Tiempo. México: Fondo de Cultura Económica.

Hernández, M. (2015): "El concepto de discapacidad: de la enfermedad al enfoque de derechos". Revista CES Derecho, 6 (2): 46-59.

Honneth, A. (2006): “Redistribución como reconocimiento”, en Fraser, N. y Honneth, A. (2006): ¿Redistribución o reconocimiento? Madrid: Morata.

Honneth, A. (1997): “Reconocimiento y obligación moral”. Areté, 9 (2): 235-252.

Izquierdo, D. et al. (2016): Nivel de sobrecarga y características de cuidado en cuidadores con trastornos mentales, Cartagena (en línea). <http://190.242.62.234:8080/jspui/bitstream/11227/3103/1/final-entregado-SUSTENTACION.pdf $>$, acceso 10 de enero de 2018.

Izquierdo, E. (2015): "Enfermería: teoría de Jean Watson y la inteligencia emocional, una visión humana". Revista Cubana de Enfermería. 31 (3).

Kohlberg, L. (1981): Philosophy of moral development: moral stages and the idea of justice. San Francisco: HarpRow Publishers. 
López, I. (2010): "El grupo de discusión como estrategia metodológica de investigación: Aplicación a un caso". Edetania, 38: 147-156.

López, J. (2013): Calidad de vida en cuidadores informales de adultos mayores con enfermedades crónicas del Hospital Nacional Guillermo Almenara Irigoyen, Perú (en línea). <http://repositorio.urp.edu.pe/bitstream/handle/urp/312/Lopez_jj.pdf?sequence=1\&isAllowed=y >, acceso 10 de mayo de 2018.

Martín, M. (2008): “Los cuidados y las mujeres en las familias”. Política y Sociedad (45) 2: 29-47.

Ministerio de Salud y Protección Social (2016): Resolución 005928 de 2016 (en línea). <https://www.minsalud.gov. co/sites/rid/Lists/BibliotecaDigital/RIDE/DE/DIJ/resolucion-5928-de-2016.pdf>, acceso 10 de enero de 2018.

Montorio, I. et al. (1998): "La entrevista de carga del cuidador. Utilidad y validez del concepto de carga". Anales de Psicología 14 (2): 229-248.

Moreno, M. y Cárdenas, A. (2010): Familia y capacidades diferentes: una oportunidad para crecer. Convenio 3188 primera infancia e inclusión social. Bogotá: Secretaría Distrital de Integración Social \& FSC.

Noddings, N. (2005): Caring in education (en línea). <http://www.uvm.edu/ rgriffin/NoddingsCaring.pdf>, acceso el 25 de noviembre de 2019 .

Organización Mundial de la Salud (2001a): Clasificación Internacional del Funcionamiento, de la Discapacidad y de la Salud. Ginebra: Organización Mundial de la Salud.

Organización Mundial de la Salud (2001b): Clasificación Estadística Internacional de Enfermedades y Problemas Relacionados con la Salud (CIE-10). Ginebra: Organización Mundial de la Salud.

Organización de las Naciones Unidas (2006): Convención sobre los derechos de las personas con discapacidad (en línea). <https://www.un.org/esa/socdev/enable/documents/tccconvs.pdf>, acceso el 12 de junio de 2019.

Orloff, A. (1996): "Gender in the Welfare States". Annual Review of Sociology, 22: 51-78.

Orloff, A. (1993): "Gender and the social rights of citizenship: The comparative analysis of gender relations and welfare states". American Sociological Review, 58: 303-328.

Palacios, P. y Jiménez, K. (2008): "Estrés y depresión en cuidadores informales de pacientes con trastorno afectivo bipolar”. Avances en Psicología Latinoamericana, 26 (2): 195-210.

Porcar, M. et al. (2015): "Cuidado familiar de la persona con trastorno mental grave: Una revisión integradora". Revista Latino-Am. Enfermagem, 23 (2): 352-360.

Sainsbury, D. (1999): Gender and Welfare State Regimes. Oxford: Oxford University Press.

Sainsbury, D. (1996): Gender, Equality and Welfares States. Cambridge: Cambridge University Press.

Sandoval, C. (1996): Investigación cualitativa (en línea). <https://panel.inkuba.com/sites/2/archivos/manual\%20 colombia\%20cualitativo.pdf>, acceso 31 de julio de 2019.

Skocpol, T. (1992): Protecting Soldiers and Mothers. Cambridge: Belknap Press.

Swanson, K. (2007): Teoría de los cuidados. Madrid: Elsevier.

Tobío, C. (2012): "Cuidado e identidad de género, de las madres que trabajan a los hombres que cuidan”. Revista Internacional de Sociología, 70 (2): 399-422.

Urra, E. et al. (2011): "Algunos aspectos esenciales del pensamiento de Jean Watson y su teoría de cuidados transpersonales”. Ciencia y enfermería, 17 (3): 11-22. 
Watson, J. (2006). Theory evolution: Watson's caring theory. Colorado (USA): University of Colorado Health Science.

Watson, J. (1979): Nursing: The philosophy and science of caring. Boston: Little Brown.

Vaquiro, R. y Stiepovich, B. (2010): “Cuidado informal, un reto asumido por la mujer”. Ciencia y enfermería, 16 (2): 9-16.

Velasco, C.L. y Sinibaldi, G.J. (2001): Manejo del enfermo crónico y su familia. México: Manual Moderno.

Velázquez, P. y Espín, A. (2014): "Repercusión psicosocial y carga en el cuidador informal de personas con insuficiencia renal crónica terminal”. Revista Cubana de Salud Pública, 40 (1) 3-17.

Zambrano, R. y Ceballos, P. (2007): “Síndrome de carga del cuidador". Revista Colombiana de Psiquiatría, 45 (1): 29-36. 